\title{
Baicalein suppresses the proliferation and invasiveness of colorectal cancer cells by inhibiting Snail-induced epithelial-mesenchymal transition
}

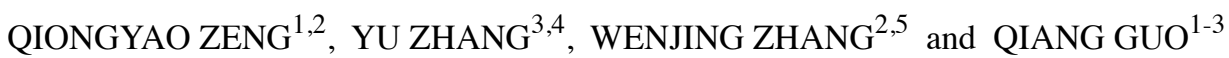 \\ ${ }^{1}$ Faculty of Life Science and Biotechnology; ${ }^{2}$ Faculty of Medicine, Kunming University of Science and Technology, \\ Kunming, Yunnan 650500; ${ }^{3}$ Department of Gastroenterology, The First People's Hospital of Yunnan Province; \\ ${ }^{4}$ Yunnan Provincial Institute of Digestive Medicine; ${ }^{5}$ Department of Medical Oncology, \\ The First Peoples' Hospital of Yunnan Province, Kunming, Yunnan 650032, P.R. China
}

Received June 7, 2019; Accepted March 6, 2020

DOI: $10.3892 / \mathrm{mmr} .2020 .11051$

\begin{abstract}
Scutellaria baicalensis (S. baicalensis) is a plant that is widely used for medicinal purposes. Baicalein, one of the primary bioactive compounds found in S. baicalensis, is thought to possess antitumor activity, although the specific mechanisms remain unclear. Therefore, the present study aimed to evaluate the ability of baicalein to disrupt the proliferation and metastatic potential of colorectal cancer (CRC) cells; a rapid and sensitive ultra-high performance liquid chromatography-tandem mass spectrometric method was employed for the identification of baicalein in an S. baicalensis aqueous extract and in rat plasma. To investigate the effects of baicalein, Cell Counting Kit-8 (CCK-8), western blotting, wound-healing and Transwell assays were performed. The data indicated that baicalein was absorbed into the blood and was able to effectively disrupt the proliferation, migration and invasion abilities of CRC cells in a dose- and time-dependent manner. Baicalein treatment was also revealed to decrease the expression of epithelial-mesenchymal transi-
\end{abstract}

Correspondence to: Professor Qiang Guo, Department of Gastroenterology, The First People's Hospital of Yunnan Province, 157 Jinbi Road, Kunming, Yunnan 650032, P.R. China

E-mail: gqkj003@sina.com

Professor Wenjing Zhang, Faculty of Medicine, Kunming University of Science and Technology, 727 South Jingming Road, Kunming, Yunnan 650500, P.R. China

E-mail: wenjing_zhang1@163.com

Abbreviations: UPLC-MS/MS, ultra-high performance liquid chromatography-tandem mass spectrometric; S. baicalensis, Scutellaria baicalensis; CCK-8, Cell Counting Kit-8; EMT, epithelial-mesenchymal transition; CRC, colorectal cancer; DMSO, dimethyl sulfoxide; OD, optical density; PBS, phosphate-buffered saline; FBS, fetal bovine serum

Key words: colorectal cancer, baicalein, Snail, epithelial-mesenchymal transition, p53, p21 tion (EMT)-promoting factors including vimentin, Twist1, and Snail, but to upregulate the expression of E-cadherin in CRC cells. The expression levels of cell cycle inhibitory proteins p53 and p21 also increased following baicalein treatment. In addition, Snail-induced vimentin and Twist1 upregulation, as well as E-cadherin downregulation, were reversed following treatment with baicalein. In conclusion, the results of the present study indicate that baicalein may suppress EMT, at least in part, by decreasing Snail activity.

\section{Introduction}

Colorectal cancer (CRC) is the third most common cause of cancer-related deaths (1), and the incidence rates are rapidly increasing, with $>1$ million new cases and 694,000 mortalities each year worldwide (2). The current first-line treatment for CRC is a combination of radiotherapy and chemotherapy (3), where drugs such as cisplatin are frequently employed (4). However, such treatments have serious side effects and are often associated with drug resistance, resulting in the constant need to identify alternative treatment options with fewer side effects. This has led to increased interest in the use of natural products to treat CRC (5).

Epithelial-mesenchymal transition (EMT) is a key process in cancer metastasis, which is characterized by the decreased expression of cell-cell adhesion molecules such as E-cadherin, and the increased expression of mesenchymal proteins such as vimentin. During EMT, epithelial cells adopt a mesenchymal phenotype and exhibit increased migratory potential; this allows for increased invasiveness and resistance to apoptosis $(6,7)$. Furthermore, EMT promotes cancer cell resistance to chemo- and radiotherapy (8). Snail is a member of the zinc-finger transcription factor family, and is reportedly one of the most important transcriptional regulators of EMT (9). Zheng et al (10), revealed that in CRC, Snail expression was significantly enhanced, which affected cancer progression. Additionally, Kwon et al (11) suggested that Snail may be a novel prognostic biomarker and therapeutic target in CRC. In fact; to an extent, the activation of Snail is considered to be the initiating factor for EMT in various malignant tumors. 
Flavonoids are a diverse family of polyphenolic compounds derived from plant-based foods, including fruit, seeds, vegetables, herbs, tea and wine. Flavonoids have been extensively studied and their anticancer effects are well documented (12). Epidemiological studies have identified that the increased intake of dietary flavonoids is associated with a decreased risk of developing CRC (13). Previous studies also revealed that flavonoids suppressed the migration and invasion abilities (14), influenced cell cycle progression (15) and induced apoptosis in CRC cells (16-18). In Asia, S. baicalensis, whose constituents include numerous flavonoid compounds, is widely used for the treatment of hypertension (19), inflammation (20) and cancer (21), as well as bacterial and viral infectious diseases (22). Baicalein, which is currently one of the most representative flavonoid aglycones in S. baicalensis, has received considerable attention for its reported ability to suppress cellular proliferation and induce apoptosis (17,23-26). However, the specific mechanisms of these anti-metastatic properties remain unclear.

In the present study, baicalein was extracted and analyzed using ultra-high performance liquid chromatography-tandem mass spectrometry (UPLC-MS/MS), and its effect on cell proliferation, migration and invasion, and the expression of EMT markers was subsequently evaluated. Notably, Snail-induced EMT was partially blocked by baicalein treatment, which provides theoretical evidence for its use as a potential antitumor agent, and indicates a novel mechanism for its antitumor effects.

\section{Materials and methods}

Chemicals. High performance liquid chromatography (HPLC)-grade methanol and formic acid were obtained from Merck KGaA. Deionized water was prepared via Milli-Q water purification (EMD Millipore). Baicalein reference standards (cat. no. 18031608) were purchased from the Beijing Aoke Biological Technology Corporation, and stock solutions were prepared in dimethyl sulfoxide (DMSO) and stored at $4^{\circ} \mathrm{C}$. Methyl p-hydroxybenzoate (internal standard; purity $>98 \%$ by HPLC-UV) was obtained from Sigma-Aldrich (Merck KGaA).

UPLC/MS/MS instrument and conditions. In order to detect and analyze baicalein in rat plasma after oral administration of $S$. baicalensis extract, UPLC-MS/MS was conducted as previously described (27). An Acquity UPLC system (Waters Corporation) and an API 4000 Triple Quadrupole mass spectrometer (Shanghai AB SCIEX Analytical Instrument Trading Co.) equipped with an electrospray ionization (ESI) source were used as a part of this system, using Analyst 1.5 software (Applied Biosystems; Thermo Fisher Scientific, Inc.) for assay control. An Acquity UPLC HSS BEH C18 column (100 x $2.1 \mathrm{~mm}, 1.7 \mu \mathrm{m}$; Waters Corporation) maintained at $40^{\circ} \mathrm{C}$ was used for chromatographic separation, with a mobile phase of (A) $0.1 \%$ formic acid, and (B) methanol, with a gradient elution of $60-90 \%$ (A) for $0-6 \mathrm{~min}, 90 \%$ (A) for 6-7 min, 90-35\% (A) for 7-10 $\mathrm{min}$, and 35\% (A) for $10-12 \mathrm{~min}$. The flow rate of the mobile phase was $0.20 \mathrm{ml} /$ min, with a $1-\mu l$ injection volume. The electrospray ionization source was performed in negative ionization mode with the following parameters: $-4.5 \mathrm{kV}$ ion spray voltage, $500^{\circ} \mathrm{C}$ turbo spray temperature. For gas pressures, nebulizer, heater and curtain gas were set to 55,50 and 25 psi, respectively, with a dwell time of $50 \mathrm{msec}$. Detection analysis was conducted in multiple reaction monitoring mode at the transition $\mathrm{m} / \mathrm{z}$ $[\mathrm{M}-\mathrm{H}]^{-} 269.2 \rightarrow 195.0$ for baicalein and $150.9 \rightarrow 136.0$ for IS, with collision energies of $-35 \mathrm{eV}$ and $-19 \mathrm{eV}$, and cone voltages of $-70 \mathrm{~V}$ and $-56 \mathrm{~V}$, respectively.

Preparation of $S$. baicalensis extract. To obtain an aqueous extraction of S. baicalensis, $100 \mathrm{~g} \mathrm{~S}$. baicalensis roots were immersed in distilled water for 30 min with occasional stirring, and then boiled three times for $30 \mathrm{~min}$ each; plant:water ratios were maintained at 1:10. The three separate decoctions were combined and concentrated into a final volume of $100 \mathrm{ml}$ to yield S. baicalensis extract, which was used for UPLC-MS/ MS analysis. The raw materials were identified by Professor Minghua Qiu of Kunming Institute of Botany, Chinese Academy of Sciences (Kunming, China), where voucher specimens are retained.

Animal study. A total of six male Sprague-Dawley rats (weight, $250 \pm 20$ g; age, 8 weeks) were provided by Kunming Medical University (Yunnan, China). The rats were housed under standard conditions $\left(20 \pm 2^{\circ} \mathrm{C}\right.$ with $60 \pm 5 \%$ humidity and 12-h light/ dark cycles) with free access to food and water, and acclimated for 1 week. Animals were observed daily throughout the study. All rats were fasted, with free access to water, for $12 \mathrm{~h}$ prior to the experiment. The rats were then randomized into 2 groups ( $=3$ per group); the experimental group received S. baicalensis extract $(4.5 \mathrm{~g} / \mathrm{kg})$ by intragastric administration once (28), and the control rats received distilled water only (10 $\mathrm{ml} / \mathrm{kg}$ of body weight). At $45 \mathrm{~min}$ after oral administration, rats were then anesthetized using pentobarbital sodium at a dose of $50 \mathrm{mg} / \mathrm{kg}$ (i.p.), and blood samples $(0.5 \mathrm{ml})$ were collected via retro-orbital bleeding. The anesthetic agent doses selected were based on existing literature (29). The sampling time-points were selected based on previous pharmacokinetic studies (27). Subsequently, the rats were sacrificed by cervical dislocation. Plasma was then separated from blood after centrifugation at $5,000 \mathrm{x}$ for $10 \mathrm{~min}$ at $4^{\circ} \mathrm{C}$. The humane endpoint of this experiment was as follows: A marked reduction in food or water intake, labored breathing, inability to stand, and no response to external stimuli. No abnormal signs that signified the humane endpoints of the experiment were observed from any of the rats during the experiment. All animal procedures were approved and performed in compliance with the guidelines set by the Animal Care Committee of the First People's Hospital of Yunnan Province (30).

Sample preparation. A total of $10 \mu 1$ IS (methyl p-hydroxybenzoate, $1 \mu \mathrm{g} / \mathrm{ml}$ in methanol) and $50 \mu 10.2 \mathrm{M} \mathrm{HCl}$ were spiked into a $100-\mu 1$ sample of rat plasma, mixed and allowed to rest for $10 \mathrm{~min}$. Next, $800 \mu \mathrm{l}$ ethyl acetate was added and mixed for $3 \mathrm{~min}$, followed by centrifugation for $5 \mathrm{~min}$ at $5,000 \mathrm{x} \mathrm{g}$ $\left(4^{\circ} \mathrm{C}\right)$. The supernatants were transferred to fresh tubes and evaporated using a nitrogen gas stream at room temperature. Any remaining residue was dissolved in $100 \mu \mathrm{l}$ mobile phase, mixed for $1 \mathrm{~min}$ by vortexing and centrifuged at $15,000 \mathrm{x} \mathrm{g}$ for $5 \mathrm{~min}$ at $4^{\circ} \mathrm{C}$. Finally, $1 \mu \mathrm{l}$ supernatant was injected into the UPLC-MS/MS system for baicalein detection. 
Cell culture. HT29 and DLD1 human colorectal cancer cell lines were obtained from Shanghai Cell Biological Institute of the Chinese Academy of Sciences, and cultured in RPMI-1640 media (Hyclone; GE Healthcare Life Sciences) containing $10 \%$ fetal bovine serum (FBS; Gibco; Thermo Fisher Scientific, Inc.) and $1 \%$ penicillin-streptomycin (Thermo Fisher Scientific, Inc.) at $37^{\circ} \mathrm{C}$ with $5 \% \mathrm{CO}_{2}$.

Cell Counting Kit-8 (CCK-8) assay. The CCK-8 assay (Beyotime Institute of Biotechnology) was used to assess the effects of baicalein on cancer cell viability. HT29 and DLD1 cells were seeded into a 96-well plate at a density of $2 \times 10^{3}$ cells/well, and cultured until complete adherence. The cells were treated with baicalein at concentrations of $0,20,40,60$, 80,100 and $120 \mu \mathrm{mol} / \mathrm{l}$ for 24,48 and $72 \mathrm{~h}$, using DMSO as a negative control. The media was then replaced with fresh media containing $10 \%$ CCK-8 solution. After a further $3 \mathrm{~h}$ of incubation, the optical density (OD) of each well was assessed using a spectrophotometer at a wavelength of $450 \mathrm{~nm}$. The inhibition rates were calculated as follows: Inhibition rate $=$ [1- (OD drug treated - OD blank)/(OD control-OD blank)] $\mathrm{x} 100 \%$. The half inhibitory concentration $\left(\mathrm{IC}_{50}\right)$ for baicalein was determined using the Logit method (31), indicating the concentration of baicalein necessary to inhibit $50 \%$ cell proliferation at a given time-point.

Wound-healing assay. HT29 cells were plated in a 6-well plate at $1 \times 10^{6}$ cells/well, and cultured to $90 \%$ confluency. A $10-\mu 1$ sterile micropipette tip was used to create a wound across the monolayer, and the cells were washed twice with sterile phosphate-buffered saline (PBS) to remove debris. The cells were treated with 10,20 , and $30 \mu \mathrm{mol} / 1$ baicalein in RPMI-1640 media without FBS, and the control group was treated with $0.05 \%$ DMSO only. Cell migration was assessed using an inverted microscope (Zeiss Axio Vert.A1), original magnification, $\mathrm{x} 10$.

Transwell invasion assay. To assess the effects of baicalein on cancer cell invasiveness, a Transwell invasion assay was performed using 24-well Transwell chambers (pore size, $8 \mu \mathrm{m}$ ), pre-coated with Matrigel ${ }^{\circledR}$ for $1 \mathrm{~h}$ at $37^{\circ} \mathrm{C}$. (BD Biosciences). Following treatment with 10,20 , and $30 \mu \mathrm{mol} / 1$ baicalein at $37^{\circ} \mathrm{C}$ for $24 \mathrm{~h}, \mathrm{HT} 29$ cells were digested with $0.25 \%$ trypsin and resuspended in serum-free RPMI-1640 medium at a density of $1 \times 10^{6} / \mathrm{ml}$, and $100 \mu \mathrm{l}$ cell suspension was added into the upper chambers. The lower chambers were filled with $500 \mu \mathrm{l}$ medium supplemented with 10\% FBS. Following incubation at $37^{\circ} \mathrm{C}$ for $24 \mathrm{~h}$, the inserts were detached and non-invasive cells were gently removed with a cotton wool swab. Invaded cells were fixed with $4 \%$ paraformaldehyde for $20 \mathrm{~min}$ at room temperature and stained with $0.1 \%$ crystal violet for $15 \mathrm{~min}$ at room temperature. Stained cells were visualized using an inverted microscope (Zeiss Axio Scope.A1) and counted in 5 randomly selected fields (magnification, $x 10$ ).

Plasmid transfection. pcDNA3.1-vector and pcDNA3.1-Snail plasmids were obtained from Shanghai GeneChem Co., Ltd., and verified by DNA sequencing. The pcDNA3.1-vector plasmids were used as the controls. HT29 and DLD1 cells ( $4 \times 10^{5}$ cells/well) were seeded into 6 -well plates with complete medium and incubated at $37^{\circ} \mathrm{C}$ for $24 \mathrm{~h}$ prior to transfection. For transient transfections, cells were transfected with $2.5 \mu \mathrm{g}$ plasmid using Lipofectamine ${ }^{\circledR} 3000$ transfection reagent (Invitrogen; Thermo Fisher Scientific, Inc.) according to the manufacturer's protocol. The cells were harvested 1-2 days after transfection for further investigation.

Western blot analysis. For western blotting, HT29 and DLD1 cells were plated at $3 \times 10^{5}$ cells/well in 6-well plates, and treated with 10,20 , and $30 \mu \mathrm{mol} / 1$ baicalein or DMSO only for 24 or $48 \mathrm{~h}$ at $37^{\circ} \mathrm{C}$. Cells were washed with PBS and lysed using ice-cold RIPA buffer (Beyotime Institute of Biotechnology) for $30 \mathrm{~min}$ to extract the total protein, which was quantified using the bicinchoninic acid assay method. The lysates were then denatured in loading buffer containing $4 \% \mathrm{SDS}$, and incubated at $95^{\circ} \mathrm{C}$ for $10 \mathrm{~min}$. In total, $50 \mu \mathrm{g}$ total protein per sample was separated by $10 \%$ SDS-PAGE gel and transferred to PVDF membranes, which were subsequently blocked for $2 \mathrm{~h}$ using 5\% non-fat dry milk in TBS and $0.05 \%$ Tween 20 . The membranes were incubated overnight at $4^{\circ} \mathrm{C}$ with primary antibodies targeted against: E-cadherin (cat. no. 14472; 1:1,000; Cell Signaling Technology, Inc.), vimentin (cat. no. 5741; 1:1,000; Cell Signaling Technology, Inc.), Snail (cat. no. 3879; 1:1,000; Cell Signaling Technology, Inc.), Twist1 (cat. no. 46702; 1:1,000; Cell Signaling Technology, Inc.), p53 (cat. no. 60283-2-Ig; 1:800; ProteinTech Group, Inc.), p21 (cat. no. 10355-1-AP; 1:800; ProteinTech Group, Inc.) and $\beta$-actin (cat. no. 4970; 1:10,000; Cell Signaling Technology, Inc.). Following primary incubation, the membranes were incubated with horseradish peroxidase-conjugated goat anti-mouse (cat. no. 6946; 1:8,000; Abcam) and anti-rabbit (cat. no. 6721; 1:6,000; Abcam) IgG $(\mathrm{H}+\mathrm{L})$ secondary antibodies for $2 \mathrm{~h}$ at room temperature. Protein bands were visualized in a darkroom using enhanced chemiluminescence reagents (New Cell \& Molecular Biotech Co., Ltd.). Protein expression was quantified using ImageJ software (v.1.48; National Institutes of Health) with $\beta$-actin as the loading control.

Statistical analysis. All experiments were performed in triplicate. Data are presented as the mean \pm standard deviation. Statistical analyses were performed using SPSS software (v.22.0; IBM Corp.). One-way ANOVA followed by Dunnett's post hoc test was used to compare the treatment and control groups. $\mathrm{P}<0.05$ was considered to indicate a statistically significant difference. All graphs were generated using GraphPad Prism software (v.5.0; GraphPad Software, Inc.).

\section{Results}

Analysis of the absorption of baicalein from S. baicalensis extract. The $S$. baicalensis herb comprises a complex mixture of different phytochemicals, and contains $>60$ chemical components. Thus, the purpose of using the UPLC-MS/MS technique was to detect and identify baicalein in rat plasma following the oral administration of S. baicalensis extract. A representative chromatogram of baicalein is presented in Fig. 1.

Baicalein suppresses DLDI and HT29 cell proliferation in vitro. The anti-proliferative activity of baicalein was assessed with a CCK-8 assay, using HT29 and DLD1 cells 
A
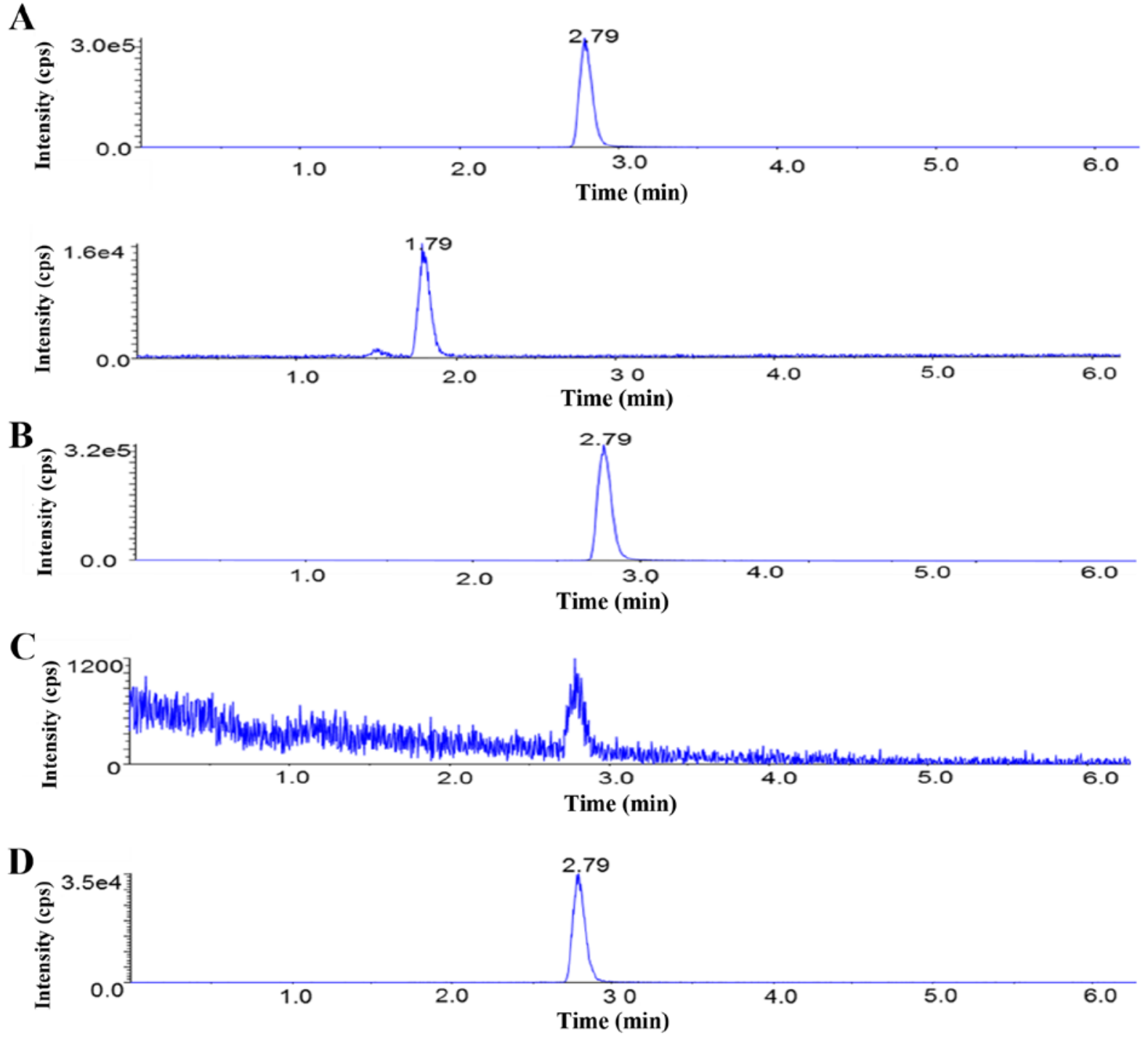

Figure 1. Ultra-high performance liquid chromatography-tandem mass spectrometric chromatogram of baicalein. (A) Baicalein and internal standard. (B) Crude S. baicalensis aqueous extract. (C) Blank rat plasma. (D) Rat plasma sample obtained 45 min after oral administration of crude $S$. baicalensis aqueous extract. S. baicalensis, Scutellaria baicalensis.

treated with $0-120 \mu \mathrm{mol} / 1$ baicalein to identify the minimal non-lethal dose. Baicalein was revealed to inhibit the viability of HT29 and DLD1 cells in a dose- and time-dependent manner; in HT29 cells, the $\mathrm{IC}_{50}$ values at 24,48 and $72 \mathrm{~h}$ were 49.77, 34.35 and $16.91 \mu \mathrm{mol} / 1$, respectively; and in DLD1 cells, the $\mathrm{IC}_{50}$ values were $60.49,34.70$, and $18.75 \mu \mathrm{mol} / 1$ at the same time-points, respectively (Fig. 2A). To avoid growth suppression, all subsequent experiments were conducted using baicalein concentrations $<34 \mu \mathrm{mol} / 1$.

p53 and p21 are reportedly involved in the baicalein-associated inhibition of CRC HCT116 cell proliferation (24). Therefore, the expression levels of p53 and p21 in HT29 and DLD1 cells were investigated, with or without baicalein treatment. As revealed in Fig. 2B and D, both p53 and p21 expression were significantly increased in baicalein-treated cells compared with the control cells, which was consistent with the aforementioned study (24).

Baicalein affects the mobility of HT29 cells in vitro. p53 is also important for the regulation of metastasis and E-cadherin expression (32). Chang et al (33), revealed that p53 inhibits the invasiveness of CRC cells by regulating EMT. To further explore the effects of baicalein on CRC cells, the migration and invasion abilities of baicalein-treated HT29 cells were investigated. The wound-healing assay results demonstrated that baicalein inhibited the migratory ability of CRC cells in a dose-dependent manner (Fig. 3A). Following treatment with 10,20 or $30 \mu \mathrm{mol} / 1$ baicalein for $48 \mathrm{~h}$, HT29 cell motility was inhibited by $49.65,67.41$ and $83.17 \%$, respectively (Fig. 3B). In the Transwell assays, cells from the control group exhibited a higher invasive capacity than those that had been treated with baicalein, indicating that baicalein significantly inhibited the invasiveness of CRC cells in a dose-dependent manner (Fig. 3C and D). These findings indicated that baicalein may act as a suppressor of CRC cell migration and invasion.

Baicalein regulates the expression of EMT markers in HT29 and DLD1 cells. EMT is an important process which is characterized by the decreased expression of epithelial markers such as E-cadherin, and the concomitant increased 
A
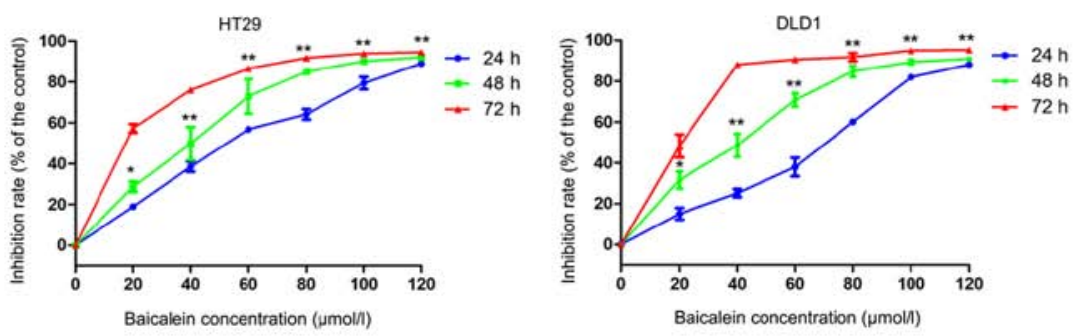

B
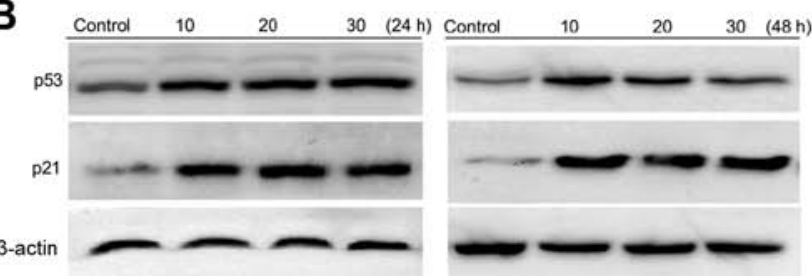

C

D
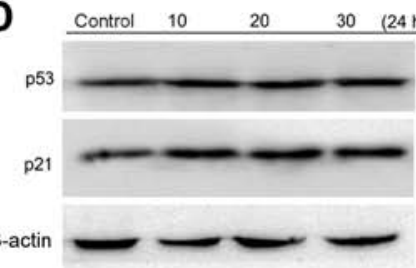
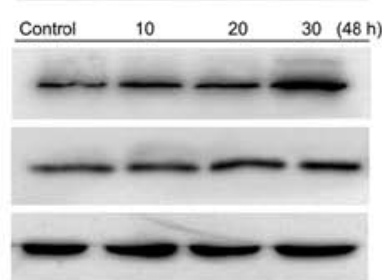
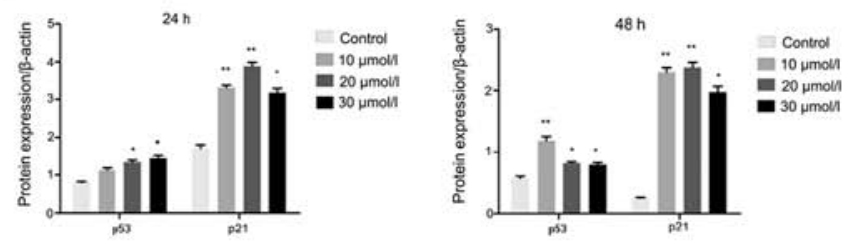

E

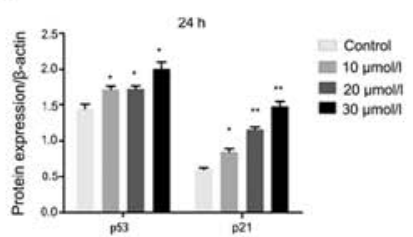

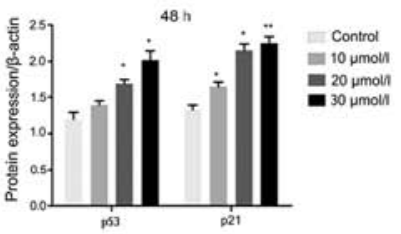

Figure 2. Baicalein suppresses cellular proliferation in CRC. (A) HT29 and DLD1 cells were treated with 0, 20, 40, 60, 80, 100 and $120 \mu$ mol/1 baicalein for 24 , 48 and $72 \mathrm{~h}$, and the CCK-8 assay was used to assess cellular proliferation. (B) Effects of baicalein on p53 and p21 expression in HT29 cells. Cells were treated with or without baicalein at the indicated concentrations for 24 or $48 \mathrm{~h}$, and the protein expression levels of p53 and p21 were determined by western blotting. $\beta$-actin was used as the internal control. (C) p53 and p21 expression in HT29 cells was quantified using ImageJ software. (D) Effects of baicalein on p53 and p21 expression in DLD1 cells. Cells were treated with or without baicalein at the indicated concentrations for 24 or $48 \mathrm{~h}$, and the protein expression levels of p53 and p21 were determined by western blotting. (E) p53 and p21 expression levels in DLD1 cells were calculated using ImageJ software. Results are expressed as the means \pm standard deviation of three separate experiments. ${ }^{*} \mathrm{P}<0.05$ and $^{* * *} \mathrm{P}<0.01$ vs. the control. CRC, colorectal cancer; CCK-8, Cell Counting Kit-8.

A

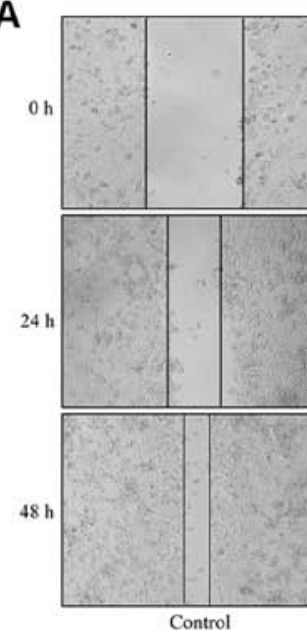

C

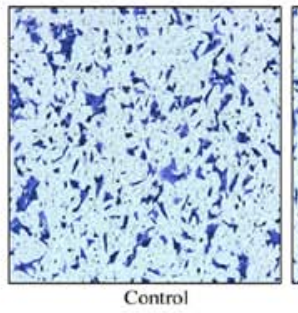

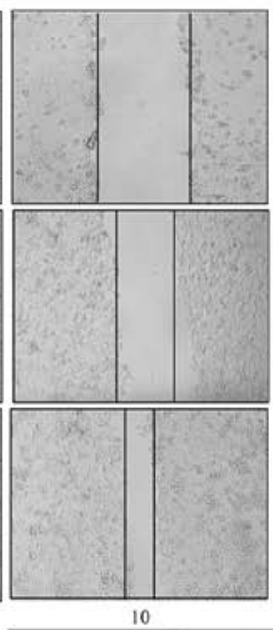
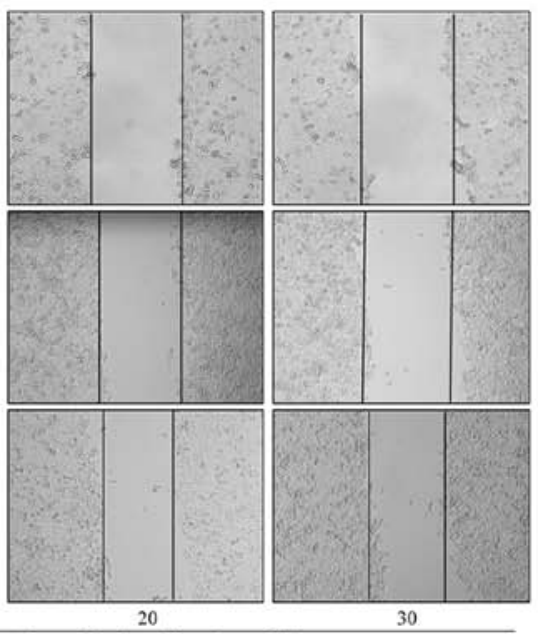

Concentration of baicalein $(\mu \mathrm{mol} / \mathrm{l})$

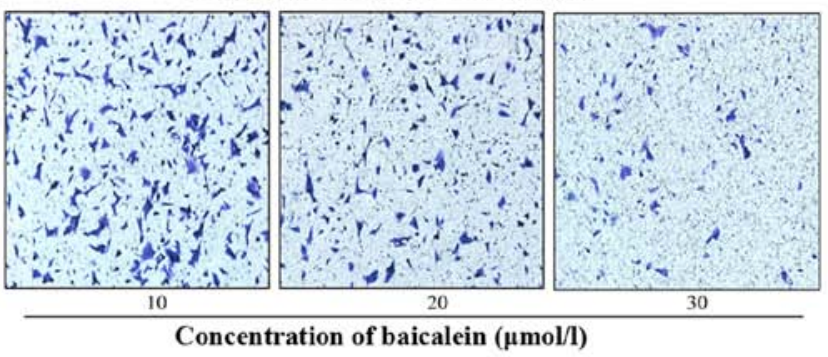

B
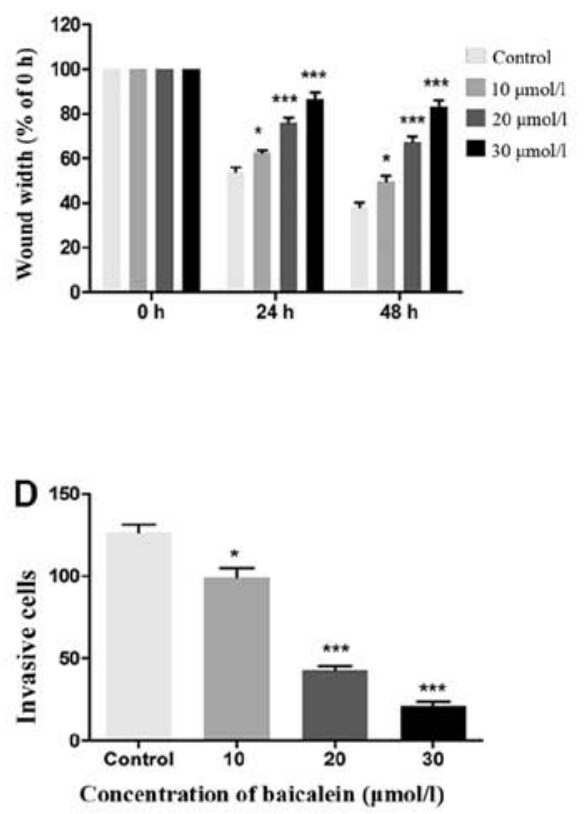

Figure 3. Baicalein inhibits cell migration and invasion in CRC. (A) Effects of baicalein on cell migration were detected using a wound-healing assay; original magnification, x10. (B) Wound width was measured to quantify the migratory potential in each group. (C) Effect of baicalein on cellular invasiveness was demonstrated by Transwell assays; original magnification, x10. (D) Invasive cells were measured to quantify the invasion potential in each group. A representative result of three independent experiments and statistical data are presented as the mean \pm standard deviation. ${ }^{*} \mathrm{P}<0.05$ and ${ }^{* * * *} \mathrm{P}<0.001$ vs. the control. CRC, colorectal cancer. 

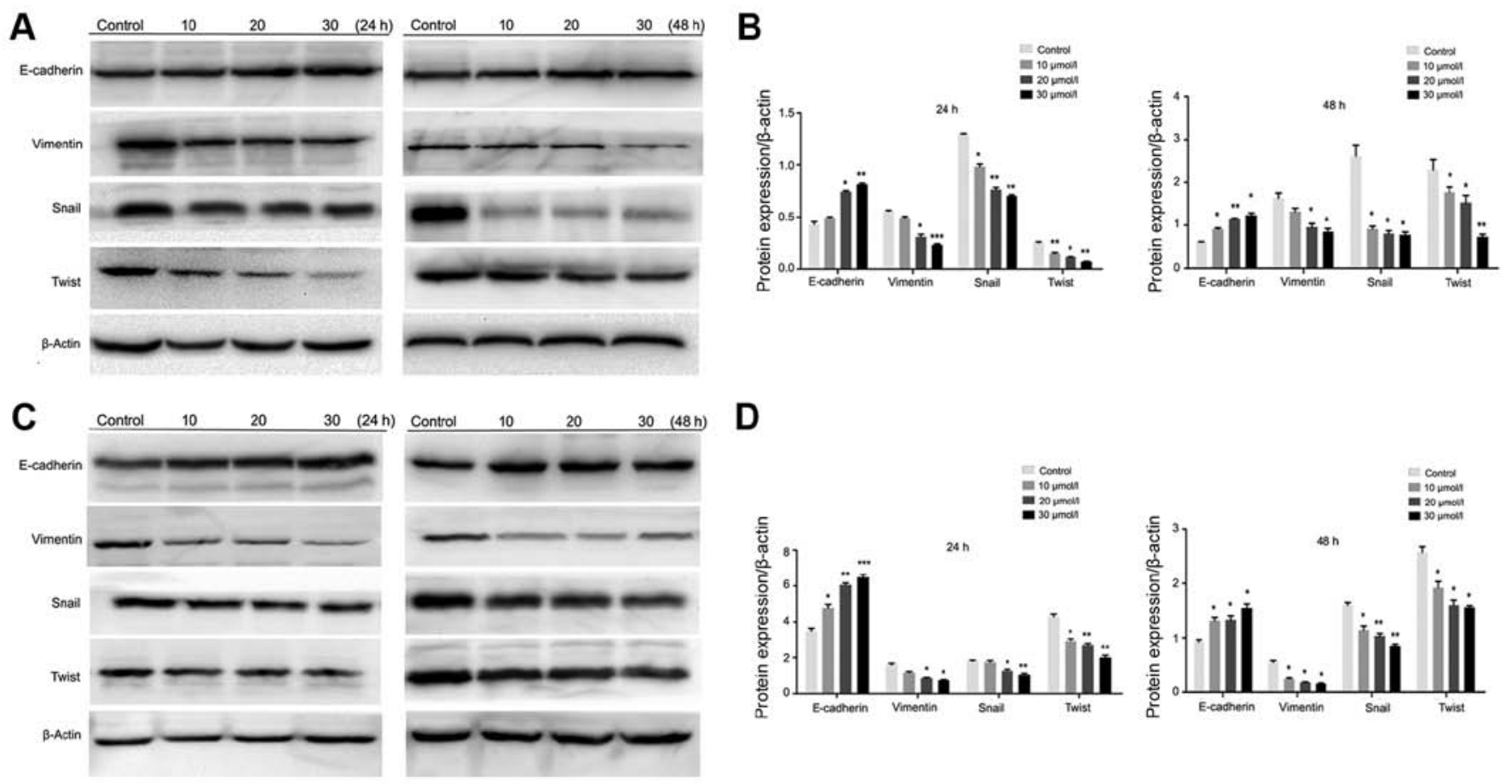

Figure 4. Baicalein regulates the expression of epithelial-mesenchymal transition-associated markers. (A) HT29 cells were treated with or without baicalein at the indicated concentrations for 24 or $48 \mathrm{~h}$, and protein expression levels of E-cadherin, vimentin, Snail and Twist1 were determined by western blotting. (B) E-cadherin, vimentin, Snail and Twist1 expression levels in HT29 cells were quantified using ImageJ software. (C) DLD1 cells were treated with or without baicalein at the indicated concentrations for 24 or $48 \mathrm{~h}$, and the protein levels of E-cadherin, vimentin, Snail and Twist 1 were determined by western blotting. (D) E-cadherin, vimentin, Snail and Twist1 expression in DLD1 cells were calculated using ImageJ software. Results are presented as the means \pm standard deviation of three separate experiments. ${ }^{*} \mathrm{P}<0.05,{ }^{* *} \mathrm{P}<0.01$ and ${ }^{* * * *} \mathrm{P}<0.001$ vs. the control. $\beta$-actin was used as the internal control.

expression of mesenchymal markers such as vimentin, matrix metalloproteinase 9 and various transcription factors (34). In the present study, the effects of baicalein on the expression of EMT markers was assessed in HT29 and DLD1 cells, following a 24 - or 48-h treatment with $0-30 \mu \mathrm{mol} / \mathrm{l}$ baicalein. Compared with the control group, Snail, Twistl and vimentin expression was decreased in baicalein-treated HT29 cells, while E-cadherin expression was increased at 24 and $48 \mathrm{~h}$, respectively (Fig. 4A and B). Similar effects were observed in DLD1 cells (Fig. 4C and D). These results indicated that baicalein was able to impede EMT in CRC cells.

Baicalein inhibits Snail-induced EMT in CRC cells. Since Snail plays a key role in metastasis and baicalein suppresses the expression of Snail and Snail-associated target genes (35), it was hypothesized that the antitumor activity of baicalein was influenced by Snail. In order to confirm this hypothesis, CRC cells were transfected with a pcDNA3.1-Snail plasmid, and Snail overexpression was confirmed by western blotting (Fig. 5A). The overexpression of Snail was revealed to increase the migratory and invasive capacities of CRC cells, which were reversed by baicalein treatment (Fig. 5B and C). Snail is reported to be one of the most important EMT-associated transcription factors; therefore, the effects of baicalein on Snail-induced EMT were also evaluated. As revealed in Fig. 5D, transfection with Snail-pcDNA3.1 markedly increased the expression levels of Snail, vimentin and Twist1, while the level of E-cadherin expression was significantly decreased, compared with the control-transfected cells; these results were consistent with the EMT expression profile. Notably, following baicalein treatment, Snail-induced vimentin and Twist1 upregulation, as well as E-cadherin downregulation were decreased both in HT29 and DLD1 cells, indicating that baicalein exhibits its suppressive effect partly through the inhibition of Snail-induced EMT.

\section{Discussion}

Plants possess a complex mixture of different phytochemicals; $S$. baicalensis contains $>60$ chemical components, of which baicalein is the primary contributor to its antitumor effects. In the present study, a UPLC-MS/MS technique was employed to detect and identify baicalein in rat plasma after the oral administration of $S$. baicalensis extract. The results demonstrate that baicalein is a major bioactive ingredient of S.baicalensis, which is absorbed into the blood via enterocytes. However, it should be noted that $S$. baicalensis is most often administered orally, and that flavonoids are usually unstable at a neutral $\mathrm{pH}$ (36). On the other hand, the flavonoid metabolite 2, 4, 6-trihydroxybenzoic acid was reported to mediate its effects through a CDK- and sodium-coupled monocarboxylate transporter 1-dependent pathway contributing to the prevention of CRC (37). Therefore, it is possible that flavonoids may be subjected to degradation by the intestinal microflora or its metabolites (38).

S. baicalensis is commonly used to treat cancer $(39,40)$; baicalein is the primary active ingredient present within extracts of $S$. baicalensis $(41,42)$. Baicalein treatment can inhibit cellular proliferation by blocking the cell cycle and 

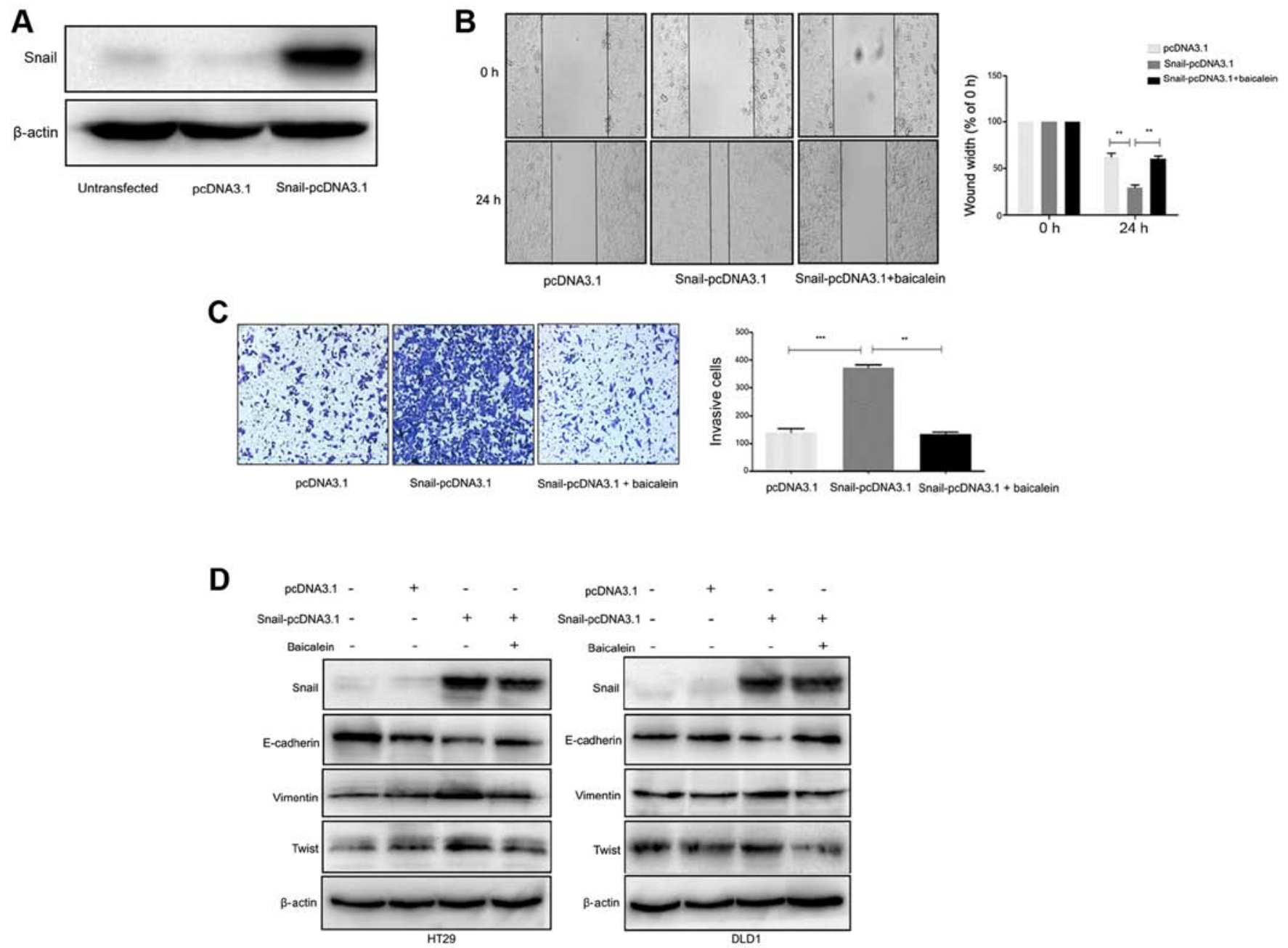

Figure 5. Overexpression of Snail reverses the inhibitory effects of baicalein on colorectal cancer cell migration and invasiveness. (A) HT29 cells were transfected with Snail-pcDNA3.1 or pcDNA3.1 and the overexpression of Snail was confirmed by western blotting. (B) Cell migration was evaluated via wound-healing assays. HT29 cells were transfected with Snail-pcDNA3.1 or pcDNA3.1. At $24 \mathrm{~h}$ post-transfection, the cells were treated with or without $30 \mu \mathrm{mol} / \mathrm{l}$ baicalein for $24 \mathrm{~h}$. Images of the wound closure were acquired; original magnification, x10; quantitative analyses are presented. Each experiment was independently repeated $\geq 3$ times. (C) Cell invasiveness was evaluated using Transwell assays. HT29 cells were transfected with Snail-pcDNA3.1 or pcDNA3.1, and at $24 \mathrm{~h}$ post-transfection, were treated with or without $30 \mu \mathrm{mol} / 1$ baicalein for a further $24 \mathrm{~h}$. The invasive HT29 cells were observed under a microscope; original magnification, x10; quantitative analyses are presented. Each experiment was independently repeated $\geq 3$ times. (D) Baicalein inhibits Snail-induced epithelial-mesenchymal transition; colorectal cancer cells were transfected with Snail-pcDNA3.1 or pcDNA3.1 for $24 \mathrm{~h}$, and then treated with or without $30 \mu \mathrm{mol} / \mathrm{l}$ baicalein for a further $48 \mathrm{~h}$. Snail, E-cadherin, vimentin, Twistl and $\beta$-actin expression levels were measured via western blotting. Images are representative of three experiments. Data are presented as the means \pm standard deviation of three independent experiments. ${ }^{* *} \mathrm{P}<0.01$ and ${ }^{* * *} \mathrm{P}<0.001$ vs. the control.

inducing apoptosis and senescence, via the modulation of the mitogen-activated protein kinase 1 ERK-1/-2, and p53-p21 pathways $(17,24)$. In the present study, baicalein exhibited its antitumor effects even at a low concentration $(10 \mu \mathrm{M})$, at which p53 and p21 expression were significantly increased. Similar results were observed for E-cadherin, vimentin, Snail and Twist 1 expression levels. It is well known that p53 induces apoptosis in response to a variety of cellular stimuli (43). Since the induction of apoptosis is a major mechanism of most chemotherapeutic agents, it was hypothesized that p53 may be involved in baicalein-mediated cellular proliferation. Notably, both p53 and p21 expression were increased in baicalein-treated cells in the present study.

Emerging evidence has revealed that EMT is responsible for the development of metastatic dissemination, a characteristic of the advanced clinical stages of CRC (44). Baicalein has been reported to inhibit EMT by regulating the Wnt/ $/$-catenin signaling pathway (45). However, the effects of baicalein on Snail, a primary promoter of EMT, have not been previously reported, to the best of the authors' knowledge. In the present study, Snail-overexpression was revealed to significantly promote CRC cell invasiveness, which was partially reversed by baicalein treatment, resulting in the downregulation of E-cadherin and the upregulation of Snail and Twist1. Hence, to the best of the authors' knowledge, the present study is the first to report that baicalein suppresses EMT, partly through a decrease in Snail activity.

To conclude, the present study confirmed that baicalein is absorbed into the blood and can inhibit cellular proliferation, migration and invasiveness in CRC, potentially by regulating p53 and p21 expression, and disrupting EMT. These data suggest that baicalein, a primary component of $S$. baicalensis, exerts potent anticancer effects against human CRC cells, and is potentially an effective target drug for cancer therapy. 


\section{Acknowledgements}

Not applicable.

\section{Funding}

The present study was supported by grants from the National Natural Science Foundation of China (grant nos. 81302159 and 81502128) and the Joint Foundation of Kunming Medical University and Yunnan Provincial Science and Technology Department (grant no. 2017FE467-159), and a grant from the Internal Division of Yunnan Provincial Health Commission (grant no. 2016NS226).

\section{Availability of data and materials}

The datasets used and analyzed during the present study are available from the corresponding author on reasonable request.

\section{Authors' contributions}

QG, WZ and QZ conceived and designed the experiments. WZ and QZ performed the experiments. YZ analyzed the data. QZ wrote the manuscript. All authors read and approved the final manuscript.

\section{Ethics approval and consent to participate}

All animal procedures were approved and performed in compliance with the guidelines set by the Animal Care Committee of the First People's Hospital of Yunnan Province.

\section{Patient consent for publication}

Not applicable.

\section{Competing interests}

The authors declare that they have no competing interests.

\section{References}

1. Friedman S, Rubin PH, Bodian C, Goldstein E, Harpaz N and Present DH: Screening and surveillance colonoscopy in chronic Crohn's colitis. Gastroenterology 120: 820-826, 2001.

2. Ferlay J, Soerjomataram I, Dikshit R, Eser S, Mathers C, Rebelo M, Parkin DM, Forman D and Bray F: Cancer incidence and mortality worldwide: Sources, methods and major patterns in GLOBOCAN 2012. Int J Cancer 136: E359-E386, 2015.

3. Pan J, Xu Y, Song H, Zhou X, Yao Z and Ji G: Extracts of Zuo Jin Wan, a traditional Chinese medicine, phenocopies 5-HTR1D antagonist in attenuating Wnt/3-catenin signaling in colorectal cancer cells. BMC Complement Altern Med 17: 506-517, 2017.

4. Li K, Guo J, Wu Y, Jin D, Jiang H, Liu C and Qin C: Suppression of YAP by DDP disrupts colon tumor progression. Oncol Rep 39: 2114-2126, 2018

5. Ling CQ, Yue XQ and Ling C: Three advantages of using traditional Chinese medicine to prevent and treat tumor. J Integr Med 12: 331-335, 2014

6. Yeung KT and Yang J: Epithelial-mesenchymal transition in tumor metastasis. Mol Oncol 11: 28-39, 2017.

7. Tsai JH and Yang J: Epithelial-mesenchymal plasticity in carcinoma metastasis. Genes Dev 27: 2192-2206, 2013.

8. Singh A and Settleman J: EMT, cancer stem cells and drug resistance: An emerging axis of evil in the war on cancer. Oncogene 29: 4741-4751, 2010.
9. Cai W, Ye Q and She QB: Loss of 4E-BP1 function induces EMT and promotes cancer cell migration and invasion via cap-dependent translational activation of snail. Oncotarget 5: 6015-6027, 2014

10. Zheng H, Shen M, Zha YL, Li W, Wei Y, Blanco MA, Ren G, Zhou T, Storz P, Wang HY, et al: PKD1 phosphorylation-dependent degradation of SNAIL by SCF-FBXO11 regulates epithelial-mesenchymal transition and metastasis. Cancer Cell 26: 358-373, 2014.

11. Kwon CH, Park HJ, Choi JH, Lee JR, Kim HK, Jo HJ, Kim HS, Oh N, Song GA and Park DY: Snail and serpinA1 promote tumor progression and predict prognosis in colorectal cancer. Oncotarget 6: 20312-20326, 2015.

12. Bugel SM and Tanguay RL: Multidimensional chemobehavior analysis of flavonoids and neuroactive compounds in zebrafish. Toxicol Appl Pharmacol 344: 23-34, 2018.

13. Bobe G, Sansbury LB, Albert PS, Cross AJ, Kahle L, Ashby J, Slattery ML, Caan B, Paskett E, Iber F, et al: Dietary flavonoids and colorectal adenoma recurrence in the Polyp Prevention Trial. Cancer Epidemiol Biomarkers Prev 17: 1344-1353, 2008.

14. Liu K, Gao H, Wang Q, Wang L, Zhang B, Han Z, Chen X, Han M and Gao M: Hispidulin suppresses cell growth and metastasis by targeting PIM1 through JAK2/STAT3 signaling in colorectal cancer. Cancer Sci 109: 1369-1381, 2018.

15. Razak S, Afsar T,Ullah A, Almajwal A, Alkholief M, Alshamsan A and Jahan S: Taxifolin, a natural flavonoid interacts with cell cycle regulators causes cell cycle arrest and causes tumor regression by activating Wnt $/ \beta$-catenin signaling pathway. BMC Cancer 18 : 1043-1061, 2018.

16. Wenzel U, Kuntz S, Brendel MD and Daniel H: Dietary flavone is a potent apoptosis inducer in human colon carcinoma cells. Cancer Res 60: 3823-3831, 2000.

17. Dou J, Wang Z, Ma L, Peng B, Mao K,Li C, Su M,Zhou C and Peng G: Baicalein and baicalin inhibit colon cancer using two distinct fashions of apoptosis and senescence. Oncotarget 9: 20089-20102, 2018.

18. Wang CZ, Calway TD, Wen XD, Smith J, Yu C, Wang Y, Mehendale SR and Yuan CS: Hydrophobic flavonoids from Scutellaria baicalensis induce colorectal cancer cell apoptosis through a mitochondrial-mediated pathway. Int J Oncol 42: 1018-1026, 2013.

19. Huang X, Wu P, Huang F, Xu M, Chen M, Huang K, Li GP, Xu M, Yao D and Wang L: Baicalin attenuates chronic hypoxia-induced pulmonary hypertension via adenosine A2A receptor-induced SDF-1/CXCR4/PI3K/AKT signaling. J Biomed Sci 24: 52-66, 2017.

20. Hong GE, Kim JA, Nagappan A, Yumnam S, Lee HJ, Kim EH, Lee WS, Shin SC, Park HS and Kim GS: Flavonoids identified from Korean Scutellaria baicalensis Georgi inhibit inflammatory signaling by suppressing activation of NF- $\varkappa \mathrm{B}$ and MAPK in RAW 264.7 cells. Evid Based Complement Alternat Med 2013: 912031-912042, 2013.

21. Hussain I, Waheed S, Ahmad KA, Pirog JE and Syed V: Scutellaria baicalensis targets the hypoxia-inducible factor-1 $\alpha$ and enhances cisplatin efficacy in ovarian cancer. J Cell Biochem 119: 7515-7524, 2018.

22. Duan C, Matsumura S, Kariya N, Nishimura M and Shimono T: In vitro antibacterial activities of Scutellaria baicalensis Georgi against cariogenic bacterial. Pediatr Dent J 17: 58-64, 2017.

23. Kim SJ, Kim HJ, Kim HR, Lee SH, Cho SD, Choi CS, Nam JS and Jung JY: Antitumor actions of baicalein and wogonin in HT-29 human colorectal cancer cells. Mol Med Rep 6: 1443-1449, 2012.

24. Chen Z, Hou R, Gao S, Song D and Feng Y: Baicalein inhibits proliferation activity of human colorectal cancer cells HCT116 through downregulation of Ezrin. Cell Physiol Biochem 49: 2035-2046, 2018.

25. Rui X, Yan XI and Zhang K: Baicalein inhibits the migration and invasion of colorectal cancer cells via suppression of the AKT signaling pathway. Oncol Lett 11: 685-688, 2016.

26. Chai Y, Xu J and Yan B: The anti-metastatic effect of baicalein on colorectal cancer. Oncol Rep 37: 2317-2323, 2017.

27. Cui XB, Qian XC, Huang P, Zhang YX, Li JS, Yang GM and Cai BC: Simultaneous determination of ten flavonoids of crude and wine-processed Radix Scutellariae aqueous extracts in rat plasma by UPLC-ESI-MS/MS and its application to a comparative pharmacokinetic study. Biomed Chromatogr 29: 1112-1123, 2015.

28. Zhang Y, Zhang $\mathrm{Z}$ and Song R: The influence of compatibility of rhubarb and radix scutellariae on the pharmacokinetics of anthraquinones and flavonoids in rat plasma. Eur J Drug Metab Pharmacokinet 43: 291-300, 2018.

29. Aoki T, Nishimura M, Kataoka H, Ishibashi R, Nozaki K and Miyamoto S: Complementary inhibition of cerebral aneurysm formation by eNOS and nNOS. Lab Invest 91: 619-626, 2011. 
30. Duan j, Song Z, Qi M, Bai X, Wang J,Zhang Y, Zou X, Guo Q and Wan P: Increased autophagy levels mediate cisplatin resistance in cisplatin-resistant cells while also rendering them vulnerable to autophagy induction. BioMed Res Int 10: 1736738-1736748, 2018.

31. Qiu Y, Li C, Wang Q, Zeng X and Ji P: Tanshinone IIA induces cell death via Beclin-1-dependent autophagy in oral squamous cell carcinoma SCC-9 cell line. Cancer Med 7: 397-407, 2018.

32. Zhang Y, Yan W and Chen X: Mutant p53 disrupts MCF-10A cell polarity in three-dimensional culture via epithelial-to-mesenchymal transitions. J Biol Chem 286: 16218-16228, 2011.

33. Chang CJ, Chao CH, Xia W, Yang JY, Xiong Y, Li CW, Yu WH, Rehman SK, Hsu JL, Lee HH, et al: p53 regulates epithelial-mesenchymal transition and stem cell properties through modulating miRNAs. Nat Cell Biol 13: 317-323, 2011.

34. Fan F, Samuel S, Evans KW, Lu J, Xia L, Zhou Y, Sceusi E, Tozzi F, Ye XC, Mani SA, et al: Overexpression of snail induces epithelial-mesenchymal transition and a cancer stem cell-like phenotype in human colorectal cancer cells. Cancer Med 1: 5-16, 2012.

35. Sang Y,Cheng C,Zeng YX and Kang T: Snail promotes metastasis of nasopharyngeal carcinoma partly by down-regulating TEL2. Cancer Commun Lond 38: 58-68, 2018.

36. Bermudezsoto MJ, Tomasbarberan FA and Garciaconesa M: Stability of polyphenols in chokeberry (Aronia melanocarpa) subjected to in vitro gastric and pancreatic digestion. Food Chem 102: 865-874, 2007.

37. Sankaranarayanan R, Valiveti CK, Kumar DR, Van slambrouck S, Kesharwani S, Seefeldt T, Scaria J, Tummala H and Bhat G: Van slambrouck S, Kesharwani SS, Seefeldt T, Scaria J, Tummala H and Bhat GJ: The flavonoid metabolite 2,4,6-Trihydroxybenzoic acid is a CDK inhibitor and an anti-proliferative agent: A potential role in cancer prevention. Cancers (Basel) 11: 2-18, 2019.

38. Gao K, Xu A, Krul C, Venema K, Liu Y, Niu Y, Lu J, Bensoussan L, Seeram NP, Heber D, et al: Of the major phenolic acids formed during human microbial fermentation of tea, citrus, and soy flavonoid supplements, only 3,4-dihydroxyphenylacetic acid has antiproliferative activity. J Nutr 136: 52-57, 2006.
39. Du LY, Qian DW, Shang EX, Liu P, Jiang S, Guo JM, Su SL, Duan JA, Xu J and Zhao M: UPLC-Q-TOF/MS-based screening and identification of the main flavonoids and their metabolites in rat bile, urine and feces after oral administration of Scutellaria baicalensis extract. J Ethnopharmacol 169: 156-162, 2015.

40. Park KI, Park HS, Kang SR, Nagappan A, Lee DH, Kim JA, Han DY and Kim GS: Korean Scutellaria baicalensis water extract inhibits cell cycle G1/S transition by suppressing cyclin D1 expression and matrix-metalloproteinase-2 activity in human lung cancer cells. J Ethnopharmacol 133: 634-641, 2011.

41. Zhang Y, Song L, Cai L, Wei R, Hu H and Jin W: Effects of baicalein on apoptosis, cell cycle arrest, migration and invasion of osteosarcoma cells. Food Chem Toxicol 53: 325-333, 2013.

42. Kowalczyk E, Krzesiński P, Kura M, Niedworok J, Kowalski J and Błaszczyk J: Pharmacological effects of flavonoids from Scutellaria baicalensis. Przegl Lek 63: 95-96, 2006 (In Polish).

43. Chao C, Saito S, Kang J, Anderson CW, Appella E and Xu Y: p53 transcriptional activity is essential for $\mathrm{p} 53$-dependent apoptosis following DNA damage. EMBO J 19: 4967-4975, 2000.

44. Yilmaz M and Christofori G: EMT, the cytoskeleton, and cancer cell invasion. Cancer Metastasis Rev 28: 15-33, 2009.

45. Liu H, Dong Y, Gao Y, Du Z, Wang Y, Cheng P, Chen A and Huang $\mathrm{H}$ : The fascinating effects of baicalein on cancer: A review. Int J Mol Sci 17: 1681-1699, 2016.

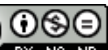

This work is licensed under a Creative Commons Attribution-NonCommercial-NoDerivatives 4.0 International (CC BY-NC-ND 4.0) License. 